\title{
RATIONALITÉ ET IDÉOLOGIE DANS LES SCIENCES DE L'HOMME
}

\author{
LE CAS DES EXPERIENCES HAWTHORNE (1924-1933) \\ ET DE LEUR REEXAMEN HISTORIQUE
}

Dans la tradition de recherches que l'on désigne couramment par l'expression de sociologie du travail (qui a supplanté en français celle de sociologie industrielle) à laquelle le lecteur non initié peut trouver une introduction à la fois dense et nuancée, d'une part, dans l'excellent ouvrage de B. Mottez ${ }^{1}$, d'autre part, dans le livre collectif Le Travail et sa sociologie. Essais critiques" ${ }^{2}$, les expériences dites "expériences Hawthorne " (1924-1933) jouent le rôle d'un véritable " moment fondateur $"{ }^{3}$. Ces expériences sont, en effet, unanimement présentées comme constituant le socle empirique solide sur lequel s'est appuyé le mouvement des relations humaines (Human relations movement) pour complèter et absorber l'Organisation scientifique du travail ou O.S.T. (équivalent français des expressions anglaises scientific management ou scientific administration $)^{4}$. En d'autres termes, pour reprendre l'expression empruntée ici même par Claude Blanckaert à George Stocking, c'est une "story" et, qui plus est, une "success story" 5 .

\footnotetext{
1. Bernard Motrez, La Sociologie industrielle, 1971, Paris, Presses universitaires de France, 1982.

2. Le Travail et sa sociologie. Essais critiques, Paris, L'Harmattan, 1985. Voir à ce sujet Jean-René Treanton, "Sur les débuts de la sociologie du travail", Revue française de sociologie, t. $27,4,1986$, p. $735-740$.

3. B. MoTtEZ, op. cit. supra n. 1 , p. 16 sq. et Le Travail et sa sociologie, op. cit. supra n. 2 , p. 119 sq., p. $215-216$.

4. B. MoTIEZ, op. cit. supra n. 1, p. 9.

5. George STOCKING Jr, "On the Limits of 'Presentism' and 'Historicism' in the Historiography of the Behavioral Sciences", Journal of the History of the Behavioral Sciences, 1965, vol. 5, 3, p. 211-218, repris dans Race, Culture and Evolution. Essays in the History of Anthropology, New York, Free Press/Londres, Collier-Macmillan Limited, 1968, p. 1-12. Cf. Claude BlanCKAERT, "Story" et "History" de l'ethnologie", Revue de synthèse, dans ce numéro, p. 451-467.
}

Revue de synthèse: IV S. Nos 3-4, juil.-déc. 1988. 
Le présent article a un double but. D'une part, il présente une synthèse des travaux historiques essentiels publiés sur les enquêtes Hawthorne ${ }^{6}$, cherchant ainsi à substituer l" « history » à la «story». Ces travaux procèdent à un réexamen critique des enquêtes qui conduit à leur dénier tout fondement empirique ${ }^{7}$. D'autre part, l'article a aussi un but épistémologique. Malgré le déni historique de leur valeur empirique (y compris, comme on le verra, des "preuves " apportées à l'appui de leur intuition centrale), ces enquêtes sont toujours considérées comme ayant eu en leur temps et, semble-t-il (mais ici l'affirmation est de moins en moins fréquente), de nos jours une grande valeur heuristique ${ }^{8}$. On se trouve donc en présence d'un paradoxe dans l'histoire du développement scientifique. Comment, en effet, une intuition se réclamant d'expériences qui la contredisent a-t-elle pu modifier l'orientation des travaux scientifiques de manière aussi radicale? Quelle est dans ce cas la relation entre croire et savoir (on pourrait ajouter au premier terme "croire savoir ») dans les sciences de l'homme? Question proche mais sensiblement différente : quelle est la part de l'idéologie et celle de la rationalité dans le savoir scientifique touchant aux sciences de l'homme?

\section{I. -- LES EXPÉRIENCES HAWTHORNE}

Hawthorne est le nom des établissements industriels de la Compagnie Western Electric situés en partie dans les faubourgs ouest de Chicago,

6. Il s'agit pour l'essentiel de: Alex Carey, «The Hawthorne Studies: A Radical Criticism ", American Sociological Review, t. 32, 3, June 1967, p. 403-417 ; Richard Herbert Franke, James D. KAUL, « The Hawthorne Experiments : First Statistical Interpretation ", American Sociological Review, t. 43, 5, oct. 1978, p. 623-643; E. SARIN, " Elton Mayo et les expériences de la Western Electric ", manuscrit non publié, 21 p. Le lecteur peut aussi se reporter à l'article postérieur de R. H. FRANKE, "The Hawthorne Experiments : Re-view ", American Sociological Review, t. 44, 1979, p. 861-867, et à Dana BramEL, Ronald FrIEND, "Hawthome, The Myth of the Docile Worker and Class Bias in Psychology ", American Psychologist, t. 36, 8, Aug. 1981, p. 867-878.

7. Henry A. LANDSBerger, dans son livre Hawthome Revisited, Ithaca, Cornell University, 1958, remarquait que la plupart des critiques des expériences Hawthorne s'en prenaient en fait à l'idéologie de MAYo (cf. infra n. 11) et de WHITEHEAD (cf. infra n. 9). R. H. Franke, et J. D. KAUL, art. cit. supra n. 6, p. 637, donnent les réferences de critiques de ce type de 1937 à 1950 . Des critiques d'un autre type, stigmatisant une tendance générale réactionnaire dans les expériences Hawthorne, dont ils donnent aussi les références, reçoivent au contraire quelque crédit d'observations signalant l'absence dans les établissements de toute activité syndicale. D. BRAMEL et R. FRIEND, art. cit. supra n. 6, expriment les mêmes critiques dans le langage marxiste orthodoxe de la lutte des classes.

8. Voir sur ce point B. Motiez, op. cit. supra n. 1, p. 16 sq.; R. H. Franke, J.D. KAUL, art. cit. supra n. 6, p. 623, n. 1 et la bibliographie très complète p. 641-643, mise à jour jusqu'en 1980 par D. BRAMEL et R. Friend, art. cit. supra n. 6, p. 877-878. 
en partie dans la ville voisine de Cicero (Illinois). Du début de novembre 1924 au 8 février 1933, soit pendant huit ans et trois mois, y ont été conduites en collaboration intime entre la Direction de la Compagnie Western Electric et des chercheurs de l'extérieur, venus d'abord du National Research Council de la National Academy of Sciences, puis, à partir du 23 avril 1927 du Department of Industrial Research, équipe partiellement autonome du Fatigue Laboratory de la Harvard Graduate School of Business Administration, une série d'expériences psychométriques, psychotechniques et psychosociologiques sur la situation de travail, d'une ampleur et d'une intensité alors inusitées et jamais égalées à ce jour.

Le compte rendu quasiment officiel des origines, et du déroulement en phases successives des enquêtes ainsi que des étapes par lesquelles est passée la réflexion des expérimentateurs, du moment crucial de leur intuition centrale, et de l'ensemble de leurs conclusions, se trouve dans l'ouvrage célèbre de F. J. Roethlisberger et William J. Dickson, Management and the Worker paru en 1939. Dickson dirigeait à Hawthorne même, au sein de la Western Electric, le Employee Relations Research Department (Département des recherches sur les relations avec le personnel), tandis que F. J. Roethlisberger, professor of Human Relations à la Harvard Graduate School of Administration, était un élève d'Elton Mayo. Un livre en deux volumes de données statistiques sur les deux phases du relay assembly test room (phases 2 et 3 dans le Schéma $n^{\circ} 1$ ci-après, p. 405) dû à T. N. Whitehead est paru en 1938 sous le titre The Industrial Worker ${ }^{9}$. Toutefois, l'auteur ne prend pas en considération les variables dépendantes les plus importantes de chacune des expériences. En raison des liens de maître à disciple qui unissent Mayo à Roethlisberger, et de l'intervention effective de Mayo dans les expériences à partir d'avril $1928^{10}$ (soit au début de la phase 2 dans le Schéma $n^{\circ} 1$, p. 405), il est utile de lire les ouvrages principaux de celui-ci ${ }^{11}$. George Caspar Homans, lui-même un de ses proches

9. Fritz J. Roethlisberger, William J. Dickson, Management and the Worker, 1939, reprod. New York, Wiley and Sons, 1964; Thomas North WHITEHEAD, The Industrial Worker, Cambridge, MA, Harvard University Press, 1938, 2 vols.

10. Outre Management and the Worker, F. J. ROETHLISBERgER est aussi l'auteur d'un ouvrage plus théorique, Management and Morale, Cambridge, MA, Harvard University Press, 1941. La date de l'intervention de Mayo à Hawthorne est donnée par E. SARIN, art. cit. supra n. 6, p. 9.

11. Elton MAYO, Democracy and Freedom, Melbourne, Macmillan, 1919; «The Human Effect of Mechanization ", Papers and Proceedings of the $42^{\text {nd }}$ Annual Meeting of the American Economic Association, t. 20, 1, March 1930, p. 156-176; The Human Problems of an Industrialized Civilization, Boston, Harvard University, Graduate School of Public Administration, 1933, $2^{\text {nd }}$ ed., 1946, paperback ed., New York, Viking, 1960; The Social 
collaborateurs, a donné des présentations classiques des expériences Hawthorne en 1941,1946 et surtout $1959^{12}$. En français, on peut se référer à Georges Friedmann ${ }^{13}$ et à Bernard Mottez, déjà cité ${ }^{14}$.

La présentation détaillée des expériences différentes qui s'enchevêtrent partiellement est inévitablement fastidieuse et rarement claire. On se référera donc aux deux schémas ci-dessous, repris et adaptés de l'article de Franke et Kaul ${ }^{15}$.

Le Schéma $n^{\circ} 2$ (p. 406) présente la séquence logique, la description et la conclusion extraites par Franke et Kaul ${ }^{16}$ de Roethlisberger et Dickson ${ }^{17}$. On voit clairement comment, centrées exclusivement sur les facteurs d'environnement dans les expériences sur l'éclairage, les recherches s'élargissent dans la phase suivante, celle du premier atelier expérimental d'assemblage de relais de téléphone (first relay assembly test room) pour examiner les effets des facteurs d'environnement, ceux des caractéristiques physiques, du style de direction et des relations sociales sur la production. Ainsi la phase du first relay experiment est-elle cruciale puisqu'elle contient, au moins en termes généraux, tous les sujets abordés dans la suite des expériences. En outre, c'est au cours de cette phase que Whitehead ${ }^{18}$ ainsi que Roethlisberger et Dickson ${ }^{19}$ présentent leur intuition d'ensemble qui guide la conception et l'interpré-

Problems of an Industrial Civilization, Boston, Harvard University, Graduate School of Public Administration, 1945. Réed. anglaise avec une importante préface de J.H. SMrrH, Londres, Routledge \& Kegan Paul, 1975; The Political Problems of an Industrial Civilization, Boston, Harvard University, Graduate School of Public Administration, 1947. Sur Mayo, cf. Lyndall Urwick, The Life and Work of Elton Mayo, Londres, Urwick, Orr and Partners, 1960.

12. George Caspar Homans, "The Western Electric Researches ", in National Research Council Committee on Work in Industry, Fatigue of Workers : Its Relations to Industrial Production, New York, Reinhold, 1941, p. 56-99; "Some Corrections ", Review of Economics and Statistics, 31, 1949, p. 319-321; The Human Group, New York, Harcourt and Brace, 1959.

13. Georges Friedmann, Problèmes humains du machinisme industriel, Paris, Gallimard, $1946,3^{e}$ ed. revue et augmentée 1954, p. 301-323. Noter en 1935 son article sur «.Frederic Winslow Taylor : l'optimisme d'un ingénieur ", Annales d'histoire économique et sociale, 36 , nov. 1935 , p. 584-602.

14. B. MotTEZ, op. cit. supra n. 1.

15. R. H. Franke, J. D. KAUL, art. cit. supra n. 6.

16. Ibid., p. 626.

17. F. J. Roethlisberger, W. J. Dickson, op. cit. supra n. 9.

18. T. N. WHITEHEAD, op. cit. supra n. 9.

19. F. J. Roethlisberger, W. J. Dickson, op. cit. supra n. 9. 


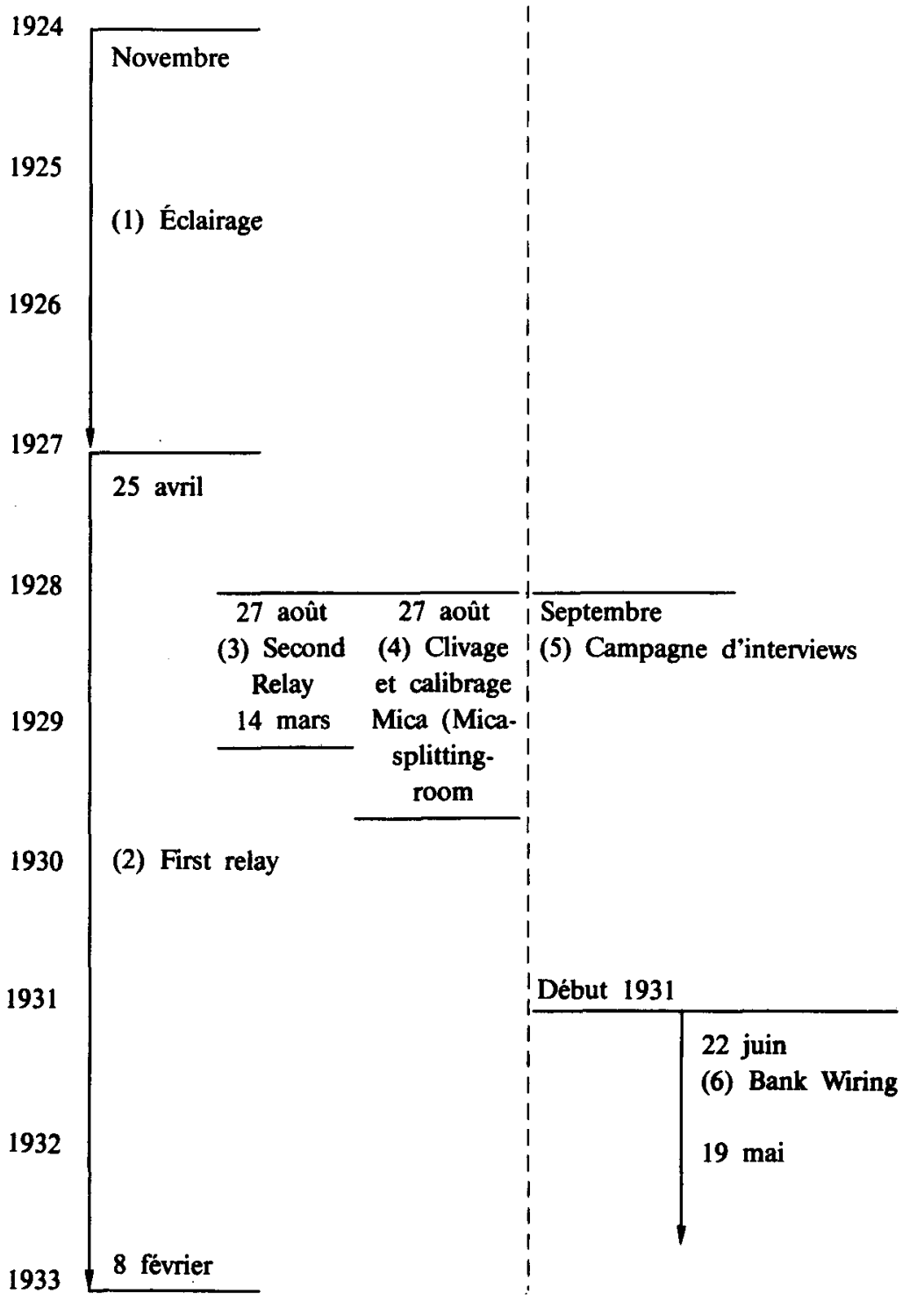

Schéma $n^{o}$ 1: Chronologie des expériences Hawthorne 
Variables expérimentales manipulées ou observées

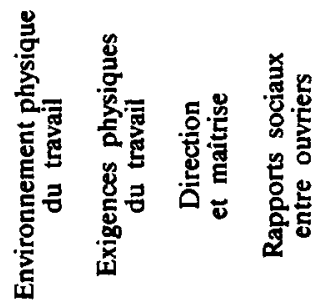

Séquence

(1) Eclaj-

$\mathbf{x}$ rage

(2)<smiles>[3H][CH]</smiles>
Relay $\mathbf{x}$ 1

(3) Second Relay

(4) Clivage du mica (Micasplitting)

(5)

campagne d'interviews

(6) Bankwiring
Description et conclusion

Trois études exploratoires suggérant que $\infty$ sont les facteurs humains plutôt que les conditions physiques de travail qui déterminent la satisfaction des ouvières et leur travail (performance).

La plus importante des expériences Hawthorne mesurant l'effet sur le travail, des pauses, du racoourcissement des périodes de travail, d'une plus grande autonomie des ouvrières, d'un stimulant financier propre au groupe expérimental. Cette étude concluait que les progrès dans le travail des ouvrières provenaient de l'amélioration des relations humaines et, à un moindre degré, des pauses.

Expérience dérivée de (2) suggérant que le stimulant financier propre au groupe expérimental n'a que des effets modérés sur le travail des ouvrières.

Expérience dérivée de (2) suggérant que les pauses et le raccourcissement des périodes de travail n'ont qu'un effet modéré sur le travail des ouvrières.

$\mathrm{x}$

Expérience dérivée de (2) renforcant les conclusions antérieures sur l'importance des interactions sociales entre ouvriers ou ouvrières et entre eux et la maîtrise. Premières indications, durant la période intensive d'interviews au début de 1931, concernant l'existence de problèmes résultant des relations entre employés, particulièrement la restriction de la production.

x Observations dérivées de (2) relevant de l'efficacité des interactions sociales au sein de groupes importants d'ouvriers dans l'établissement de standards concernant le rythme de travail (restriction de la production durant la période de la récession économique).

Schéma $n^{\circ} 2$ : Séquence logique, description et conclusion des expériences Hawthorne. 
tation de toutes les expériences. Cinq ouvrières rassemblées de leur plein gré dans cet atelier expérimental ont subi différentes modifications dans leurs conditions matérielles de travail : nombre et durée des pauses, diminution de la durée quotidienne et hebdomadaire du travail, etc. Une élévation du rendement s'ensuit, qui persiste d'après Roethlisberger et Dickson après le retour à la situation initiale. Les auteurs (qui s'appuient sur quelques déclarations des ouvrières) concluent que le changement favorable est dû aux relations agréables et confiantes régnant dans l'atelier, non seulement entre les ouvrières, mais entre ces dernières et la maîtrise (cette dernière avait gardé apparemment toutes ses prérogatives, comme on le voit, dans le remplacement de deux des ouvrières de la first relay assembly test room) ${ }^{20}$.

La relation classique de ces expériences, parue six ans après la fin de la dernière phase sous la signature de Roethlisberger et Dickson ${ }^{21}$, contient à peu près tous les thèmes qu'abordera par la suite l'école des relations humaines : motivation, moral, groupe informel, leadership, résistance au changement.

Il faut ajouter, pour compléter la description, que des programmes non expérimentaux tels que le "counseling ", la formation de la maitrise, d'autres encore, ont été entrepris par la Western Electric pour mettre en application les conclusions des six expériences.

\section{II. - LE REEXAMEN HISTORIQUE DES EXPERIENCES HAWTHORNE}

De manière surprenante pour des travaux qui occupent une place aussi centrale dans l'histoire des sciences sociales du travail en général et de la sociologie du travail en particulier, les expériences Hawthorne n'ont été étudiées sérieusement avec suffisamment d'esprit critique et de rigueur qu'à une date relativement récente, comme le note Alex Carey lui-même ${ }^{22}$. Les premières critiques, fort nombreuses, ont porté essentiellement, comme le remarquait déjà Landsberger ${ }^{23}$, sur l'idéologie de Mayo et de Whitehead.

La première analyse sérieuse du corpus des textes publiés en relation avec les enquêtes Hawthorne, et d'abord naturellement du livre de

20. T. N. WHITEHEAD, op. cit. supra n. 9, p. 111 sq.

21. F. J. Roethlisberger, W. J. Dickson, op. cit. supra n. 9.

22. A. CAREY, art. cit. supra n. 6, p. 403-404.

23. H. A. LANDSBERGER, op. cit. supra n. 7. 
Roethlisberger et Dickson ${ }^{24}$ est celle d'Alex Carey. Elle constitue ce qu'on peut appeler un réexamen empirique des données qualitatives. Richard Herbert Franke et James D. Kaul ${ }^{25}$ ont confirmé les conclusions de Carey en poussant l'analyse plus loin grâce à d'autres bases. Ils ont eu accès aux documents statistiques originaux conservés aux établissements Hawthorne, enregistrant sur une base horaire et hebdomadaire la production des vingt-neuf ouvrières et ouvriers ayant participé aux expériences, pendant huit ans et trois mois. Leur étude forme ce qu'on peut appeler un réexamen empirique des données quantitatives. Plus récemment, D. Bramel et $R$. Friend ${ }^{26}$ reprenant la perspective à la fois de Franke et Kaul et surtout de Carey mais dépassant la critique interne, adoptent un point de vue expressément marxiste pour critiquer les enquêtes Hawthorne. On peut qualifier cette démarche de réexamen qualitatif externe ou réexamen théorique. Toutefois, nous nous bornerons aux deux premières démarches.

\section{Le réexamen empirique des données qualitatives}

Carey remarque à juste titre, avec beaucoup de commentateurs, que le "grand éclaircissement [...] tout différent de ce qu'ils [les enquêteurs] avaient attendu des études sur l'éclairage " 27 s'est produit de façon fortuite pour les expérimentateurs environ un an après le début de la phase (2). Il s'agit, bien entendu, de l'importance prédominante des relations humaines par rapport aux aspects physiques et économiques de la situation de travail, seuls étudiés jusque-là. Ce sont les relations humaines qui s'avèrent les déterminants les plus importants du comportement au travail et plus précisément de la production. Les expériences (3) et (4) ont pour but explicite de clarifier et de consolider cette intuition.

L'analyse de Carey demeure insurpassable sur au moins deux points qui visent l'ensemble de la démarche de Roethlisberger (et donc de Mayo) et de Dickson :

a) La procédure "scientifiquement illettrée ".

Le premier point concerne la démarche scientifique d'ensemble suivie par les auteurs de Management and the Worker qu'il qualifie placidement de "scientifiquement illettrée ${ }^{28}$. Une telle procédure, constamment

24. F. J. Roethlisberger, W. J. Dickson, op. cit. supra n. 9.

25. R. H. Franke, J. D. KAUL, art. cit. supra n. 6.

26. D. BRAMEL, R. FRIEND, art. cit. supra n. 6.

27. F. J. Roethlisberger, W. J. DiCKSON, op. cit. supra n. 9, p. 15.

28. A. CAREY, art. cit. supra n. 6 , p. 406 , n. 17: " The scientifically illiterate procedure of dismissing non-preferred explanations..." 
utilisée par les auteurs, consiste d'après lui en un rejet des hypothèses qui n'ont pas la faveur des enquêteurs sur les bases suivantes : 1) absence de preuve décisive en leur faveur; 2) absence de preuve que l'une quelconque d'entre elles puisse à elle seule expliquer dans sa totalité l'effet que l'on a observé. Par contre, cette procédure n'est jamais appliquée aux hypothèses qui ont la préférence des enquêteurs. Celles-là sont considérées comme fondées sur un critère simple : les preuves qui leur sont contraires ne permettent pas de tirer de conclusion nette (" are less than conclusive " $)^{29}$.

b) L'expérience (2) : nature et environnement physique du travail.

Carey décèle ce travers particulièrement dévastateur dans plusieurs moments décisifs de l'argumentation de Roethlisberger et Dickson. Dès la présentation et la discussion de l'expérience (2), c'est-à-dire le first relay assembly test room, le raisonnement "biaisé " (pour ne pas dire spécieux) peut être décelé. Lorsque les enquêteurs, au terme des deux années d'expérience, constatent une augmentation de la production de $30 \%$ (vraisemblablement en volume total), ils ont déjà reçu leur fameuse " illumination » et sont donc convaincus que l'augmentation constatee provient principalement d'un changement " mental " chez les ouvrières résultant lui-même d'un changement dans les méthodes d'encadrement ${ }^{30}$.

Toutefois, les changements introduits les uns après les autres étaient nombreux, bien qu'aucun groupe de contrôle n'ait été constitué, ce qui ne laisse pas de surprendre ${ }^{31}$. L'expérience comportait, en effet, moins de variations dans les relais téléphoniques à assembler ${ }^{32}$, moins d'heures de travail, des pauses, un encadrement plus. souple et plus amical, et un stimulant financier préférentiel (calculé sur la base de la production du groupe expérimental et non du département tout entier) ${ }^{33}$. Malgré le caractère cumulatif des changements introduits et l'absence déjà mentionnée de groupe de contrôle, les expérimentateurs s'attendaient au départ à recueillir des informations sur l'influence de telle ou telle condition physique du travail ${ }^{34}$. Bien qu'ayant acquis leur certitude sur la prééminence des relations humaines, ils n'en retiennent pas moins, à titre d'hypothèse, l'idée selon laquelle l'un quelconque des changements introduits cumulativement puisse à lui seul avoir provoqué à la fois le changement d'attitude observé chez les ouvrières et l'augmentation

\footnotetext{
29. Ibid.

30. F. J. Roethlisberger, W. J. Dickson, op. cit. supra n. 9, p. 189-190.

31. Les groupes de contrôle étaient, semble-t-il, déjà une pratique courante au moins en agronomie et en psychologie.

32. F. J. Roethlisberger, W. J. Dickson, op. cit. supra n. 9, p. 87.

33. Ibid., p. 22, 30-73.

34. Ibid., p. 86-89.
} 
de la production qui lui est associée. Les hypothèses envisagées par les auteurs du rapport faisant autorité (Roethlisberger et Dickson ${ }^{35}$ ) mentionnent comme sources possibles "d'hypothèses expliquant les changements majeurs" dans le comportement au travail $\left.{ }^{36}: 1\right)$ les changements dans la nature et l'environnement physiques du travail; 2) la réduction de la fatigue et de la monotonie consécutive à l'introduction de pauses ${ }^{37}$; 3) le nouveau système de rémunération; et 4) le nouveau type d'encadrement et les changements consécutifs dans les relations humaines. Tout l'article de Carey est consacré à l'examen critique des preuves et des arguments avancés par les enquêteurs pour conclure à chaque stade en faveur de la dernière hypothèse.

Les résultats de l'enquête (4) destinée à disqualifier l'importance du stimulant financier sont pour le moins confus. Les $15 \%$ initiaux de hausse de la production ne s'observent que si l'on adopte pour base le taux de production horaire par heure de travail par semaine. Si l'on adopte la production totale hebdomadaire, on obtient au contraire une réduction de la production. Les enquêteurs soutiennent néanmoins, sur une base indéterminée, qu'il y a eu initialement dans l'expérience (4) $15 \%$ d'augmentation de la production (nous savons qu'il s'agit nécessairement de taux horaire). Ils les soustraient des 30 \% d'augmentation de la production constatée lors de l'expérience (1) (il s'agit là pratiquement de toute la production totale). Cela leur permet de conclure que les $15 \%$ restant représentent " le maximum (dans l'augmentation de la production) qui puisse être imputé à l'introduction d'un stimulant financier ${ }^{38}$ réalisée dans l'expérience (2) ". Tout en reconnaissant le caractère entièrement fictif d'un tel calcul, ils n'en poursuivent pas moins leur raisonnement jusqu'à soutenir dans un rappel des événements qu'ils ont enregistrés que :

«les test room studies viennent jusqu'à présent à l'appui de la conclusion selon laquelle [...] il est impossible de considérer [un stimulant financier] comme une chose existante en elle-mème et exerçant un effet propre sur un individu donné ${ }^{39}$.

Carey souligne à bon escient le caractère peu valide des inférences ainsi établies. En ce qui concerne la comparaison entre l'expérience (2) et l'expérience (4), il montre comment une inférence plus plausible pourrait être établie. L'expérience (4) aboutit en fait à une hausse du

35. Ibid.

36. Ibid.

37. Ibid.

38. Ibid., p. 160 .

39. Ibid. 
taux horaire de production moins que proportionnelle à la baisse de la production totale, dans une période durant laquelle le nombre d'heures de travail hebdomadaire est passé de $551 / 2$ à $461 / 6$. On pourrait simplement en conclure que toute durée du travail hebdomadaire excédant 48 heures est de peu d'utilité - ou d'une utilité nulle -, constatation fermement établie déjà depuis de nombreuses années $\mathbf{4 0}^{0}$. Il aurait été possible alors d'attribuer tout simplement les $30 \%$ d'augmentation de la production de l'expérience (2) à l'effet du stimulant financier préférentiel. Au contraire, par l'obstination dans le raisonnement et les argumentations très particulières dont on a pu donner quelques aperçus, les enquêteurs sont parvenus à la conclusion selon laquelle l'effet d'un stimulant financier est tellement soumis à l'effet des facteurs sociaux qu'il est impossible d'admettre qu'il puisse exercer le moindre effet par lui-même.

Il en va de même pour l'expérience (3), destinée comme on l'a vu à évaluer directement l'impact du stimulant financier préférentiel. Il y eut un changement important dans la production (12\% de hausse immédiate) et dans le comportement, puisque les ouvrières du département ont demandé à bénéficier des mêmes conditions de salaire. Logiquement tout semble pousser à conclure à l'importance d'un stimulant financier privilégié. Ce n'est pas la conclusion qui est adoptée.

Tout au début de leur interprétation des résultats de l'expérience (3), Roethlisberger et Dickson remarquent, après coup en quelque sorte, que figure quelque part dans le "compte rendu quotidien " (daily historic record) du groupe d'ouvrières de l'expérience (2) la mention d'une remarque de l'une des ouvrières de ce groupe, d'après qui les ouvrières du groupe de la nouvelle expérience (3) " "s'intéressaient vivement" à leur production (NB : celle des ouvrières du groupe de l'expérience (2) " ${ }^{41}$. A ce point, les auteurs se contentent de cette notation en laissant entendre qu'ils lui donnent un sens. Après vingtquatre pages, on nous dit que :

«bien que la production ait augmenté en moyenne de $12 \%$ dans l'expérience (3) il était tout à fait évident que d'autres facteurs que le changement de stimulant financier avaient contribué à cette augmentation. [...] Il y avait des preuves indiquant que les ouvrières (participant à l'expérience (3) avaient saisi dans ce test une occasion de prouver à tout

40. A. CAREY, art. cit. supra n. 6, cite un livre antérieur aux expériences Hawthorne, Horace M. VERNON, Industrial Fatigue and Efficiency, Londres, Dutton, 1921, d'après lequel dans les arsenaux une rétuction de la semaine de travail de 66 heures à 48 heures 56 (soit environ $26 \%$ ) s'accompagne d'une hausse du taux horaire de production de $68 \%$ et de la production hebdomadaire de $15 \%$.

41. F. J. Roethlisberger, W. J. Dickson, op. cit. supra n. 9, p. 134. 
un chacun qu'elles pouvaient faire aussi bien que celles de l'expérience (2). Elles étaient parties pour égaler leur score. Considérant cela, même l'évaluation la plus généreuse estimerait la hausse de production résultant du seul changement dans le mode de paiement à quelque chose de moins que. $12 \%{ }^{42}$.

Carey signale qu'en l'absence de toute preuve supplémentaire, ce jugement n'est pas sérieusement fondé.

Vers la fin de l'ouvrage (p. 577) le sujet est repris et, sans plus d'informations ni de preuves, on laisse à entendre au lecteur que la hausse de la production lors de l'expérience (3) était due à certaines "conséquences sociales " de la "situation sociale fondamentale " (" basic social situation "). On affirme simplement qu'il s'agissait d'une situation où « la rivalité (avec le groupe de l'expérience (2) fut mise en pleine lumière [" brought to a focus"] par la constitution du groupe de l'expérience (2) dont "la production s'est accrue rapidement" en conséquence $》$.

Rappelons cependant que l'introduction du stimulant financier préférentiel dans l'expérience (2) a provoqué immédiatement une hausse de la production de $12 \%$ et que sa suppression a entraîné une baisse immédiate de $17 \%$. A première vue, comme le dit $\mathrm{Carey}^{43}$, de tels résultats n'encouragent guère à minimiser l'importance des stimulants financiers ni à douter de leur capacité d'exercer des " effets propres". Néanmoins, ces résultats plutôt embarrassants ont été non seulement expliqués mais convertis en preuves à l'appui de conclusions de cet ordre, le tout sur la base d'un seul commentaire rapporté par une seule ouvrière.

\section{L' "attitude amicale de la maîtrise" ("friendly supervision") réexaminée de près dans son fonctionnement effectif}

La conclusion des expériences Hawthorne consiste, comme on l'a vu, à prétendre que l'attitude amicale de la maîtrise et les relations sociales chaleureuses qui en résultent au sein du groupe de travail sont de très loin les facteurs les plus importants affectant le comportement au travail.

L'expérience (2) aurait dû commencer sous les meilleurs auspices, puisque les cinq ouvrières avaient été choisies à la fois pour leur

42. Ibid., p. 158.

43. A. CAREY, art. cit. supra n. 6, p. 409. 
compétence et pour leur bonne volonté et leur détermination à coopérer ${ }^{44}$. On doit remarquer, toutefois, que si Whitehead mentionne lui aussi la "considerable experience in the assembly of this kind of relay" des ouvrières choisies, il estime que la méthode adoptée pour opérer le choix n'obéissait à aucune règle précise ("quite informal ") et demeurait de ce fait entachée d'obscurité. Il semble que ce soit les ouvrières elles-mêmes qui aient fait le choix en accord avec leur chef d'atelier ${ }^{45}$.

Cependant dès le tout début de l'expérience, les ouvrières se livrent entre elles à des conversations d'une telle durée qu'elles représentent un véritable problème parce qu'elles « entrainent un manque d'attention au travail et dénotent une préférence pour de longues conversations entre elles " ${ }^{46}$. Le texte de Roethlisberger et Dickson qui constitue le rapport officiel sur l'ensemble des enquêtes mentionne pour la première fois le degré de gravité atteint par le phénomène à la date du 2 août 1927, trois mois après l'installation des ouvrières dans la pièce spécialement équipée du test room. Ce jour-là, quatre des cinq ouvrières furent conduites devant le contremaître (foreman) et réprimandées pour leur excès de bavardage ${ }^{47}$. Il convient de rappeler ici le double encadrement du first relay assembly test room. Il comportait, d'un côté, l'observateur (observer) chargé d'une double fonction : tenir un relevé exact (accurate record) des observations et susciter et entretenir une atmosphère amicale dans la salle d'expérience : c'est à lui qu'incombait la majeure partie de l'encadrement quotidien; de l'autre, le contremaître, qui continuait de superviser la comptabilité, la révision des taux (de production et de rémunération), l'avancement, etc. D'août à novembre 1927, rien de spécial n'est tenté pour supprimer ce privilège bien que le contremaître ait tenté plusieurs fois de restreindre ce qu'il considérait comme du bavardage abusif. Les ouvrières désignées par Roethlisberger et Dickson comme $1 \mathrm{~A}$ et $2 \mathrm{~A}$ se distinguaient par l'absence chez elles de cette "coopération sincère" (wholehearted cooperation) souhaitée par les enquêteurs. Aux alentours du 17 novembre, la situation ne s'étant pas améliorée, on eut recours à des mesures d'ordre disciplinaire. On exigea des ouvrières qu'elles fassent un appel à chaque faute commise dans l'assemblage et on les empêcha de parler entre elles. Mais aux alentours de décembre, le manque de coopération de certaines des ouvrières devenait un sérieux sujet d'inquiétude pour

\footnotetext{
44. F. J. Roethlisberger, W. J. Dickson, op. cit. supra n. 9, p. 21.

45. T. N. WHItEHEAD, op. cit. supra n. 9, vol. I, p. 14.

46. F. J. Roethlisberger, W. J. Dickson, op. cit. supra n. 9, p. 53.

47. Les contremaittres (foremen) exerçaient à égalité avec les chefs de département et se situaient à quatre niveaux au-dessus des ouvrières.
} 
quelques-uns des membres de la direction immédiatement concernés. Les surveillants reçurent la consigne d' " alerter " les ouvrières en leur indiquant qu'elles n'obtenaient pas les résultats escomptés et que, si la production ne se redressait pas, elles perdraient leur avantage d'avoir des lunches servis gratuitement ${ }^{48}$.

Finalement les ouvrières $1 \mathrm{~A}$ et $2 \mathrm{~A}$, après des réprimandes continuelles et même quotidiennes, et des démonstrations d'autorité surtout contre l'ouvrière $2 \mathrm{~A}$, réprimandée par les autorités de la test room et même par le directeur de division (superintendent) ${ }^{49}$, jugées par les enquêteurs comme dépourvues de l' " attitude correcte ", furent congédiées de la test room pour « insubordination caractérisée " (gross insubordination), et production en baisse ou sans amélioration (static) ${ }^{50}$. Whitehead déclare quant à lui : « elles furent retirées [de la test room] du fait de leur manque de coopération, qui aurait exigé sans cette décision des sanctions beaucoup plus lourdes ${ }^{51}$.

Il est intéressant de comparer le récit de Mayo avec celui de Roethlisberger et Dickson. En 1946 Mayo dit des deux ouvrières les plus rétives qu'elles ont "abandonné " ${ }^{52}$, et, en 1949, qu'elles se sont " retirées " 33 . Il donne, en outre, en 1946 une description idyllique de l'expérience (2) citée par Carey ${ }^{54}$, qui se termine sur cette sentence assez cruelle a posteriori à son égard : «A aucun moment [durant toute l'expérience] les ouvrières n'ont eu l'impression qu'elles travaillaient sous la contrainte [feel that they were working under pressure]" ${ }^{55}$.

Étant donné les manifestations d'autorité qui ont marqué cette expérience, on comprend que Carey juge nécessaire d'établir un nouveau bilan en essayant de mettre en rapport les pratiques d'encadrement et les variations de la production. Cette dernière étant toutefois rapportée de façon lacunaire, on reprendra seulement ses conclusions.

1) A part un accroissement peu certain de 4 à $5 \%$ consécutif à l'introduction du stimulant financier préférentiel, il n'y a aucune hausse de la production hebdomadaire pendant les neuf premiers mois, malgré les égards et les avantages procurés aux ouvrières.

2) A partir du moment où commence ce que Roethlisberger et

48. T. N. WHITEHEAD, op. cit. supra n. 9, vol. I, p. 16.

49. F. J. Roethlisberger, W. J. Dickson, op. cit. supra n. 9, p. 55. Les Superintendent dirigeaient une branche des établissements et se trouvaient situés à sept niveaux au-dessus des ouvrières.

50. Ibid., p. 53-57.

51. T. N. WHITEHEAD, op. cit. supra n. 9, vol. I, p. 118.

52. E. MAYo, The Human Problems..., op. cit. supra n. 11, p. 56.

53. ID., The Social Problems..., op. cit. supra n. 11, p. 62.

54. A. CAREY, art. cit. supra n. 6, p. 411 , n. 48.

55. E. MAYo, The Human Problems..., op. cit. supra n. 11, p. 68-69. 
Dickson appellent «l'expérience proprement dite ", c'est-à-dire l'introduction du nouveau stimulant financier, il n'y a aucun accroissement de la production hebdomadaire pendant six mois. Lorsqu'il est clair que le style d'encadrement ("free and friendly supervision") n'obtient pas les résultats escomptés, on resserre la discipline, ce qui conduit au renvoi de deux ouvrières.

3) La production hebdomadaire ne connait une augmentation sensible et soutenue que lorsque les deux ouvrières les moins productives sont renvoyées et remplacées par des leaders choisies pour leur rapidité et leur capacité de production. Ces leaders prennent en main la discipline du groupe. En outre, c'est à elles que revient la majeure partie de l'augmentation de la production du groupe, en taux et en volume, pour les dix-sept derniers mois de l'expérience.

4) Après le changement d'ouvrières et l'augmentation qui s'ensuit dans la production, le style d'encadrement redevient amical (friendly) et relaxed. Mais les enquêteurs n'apportent aucune preuve permettant de conclure que la production a augmenté grâce au style d'encadrement plutôt que l'inverse. Il est clair, en tout état de cause, que le style d'encadrement « libéral » s'est traduit pour les ouvrières par un résultat très tangible, sous la forme du paiement d'heures de travail non effectuées : c'était en fait une augmentation du taux des pièces.

Carey conclut à bon droit sur l'impossibilité de généraliser toute conclusion sur la base des données publiées des trois premières expériences Hawthorne pour trois raisons : l'absence de tout échantillon représentatif d'une population plus importante que les groupes étudiés eux-mêmes; l'absence de groupe de contrôle pris par exemple dans les ateliers environnants et de données de production les concernant; l'extrême faiblesse $(\mathrm{N}=5)$ des effectifs interdisant des résultats statistiquement fiables (ce dernier point est discutable comme on le verra plus loin). A la relecture des descriptions, des incidents et des données quantitatives de Roethlisberger et Dickson, il lui semble que, de façon d'ailleurs surprenante pour lui, ces expériences viennent plutôt à l'appui de notions apparemment vieillies telles que la valeur des stimulants monétaires, du leadership énergique, et de la discipline. En recourant aux séries quantitatives directement enregistrées et conservées dans les archives des établissements Hawthorne, et en leur appliquant un traitement statistique, Richard Herbert Franke et James D. Kaul renforcent la conclusion d'Alex Carey et font une critique encore plus radicale. 


\section{III. - LE REEXAMEN EMPIRIQUE QUANTTTATIF : FRANKE ET KAUL}

Les deux auteurs soulignent d'emblée que les conclusions des expériences Hawthorne ont donné naissance à de nombreuses recherches toutes centrées sur le thème général de la satisfaction au travail (qu'il s'agisse de travaux sur la maîtrise, sur l'autoritarisme, l'organisation informelle, le leadership, le participative management, etc.) ${ }^{56}$. Or, l'objectif initial des enquêtes Hawthorne était d'expliquer les fluctuations de la production et non la satisfaction au travail. En outre, les auteurs font remarquer, ce qui accroît leur doute sur le mouvement dit des " relations humaines ", qu'il n'y a jamais eu avant eux d'analyse statistique digne de ce nom des données produites par les expériences Hawthorne. L'absence d'analyse statistique peut cependant s'expliquer par la nature même des expériences : 1) il n'y avait pas de groupe de contrôle, à l'exception, si l'on veut, des groupes expérimentaux euxmêmes avant les manipulations ; 2) il s'agissait d'expériences sur le terrain effectuées sur de longues périodes : il était donc difficile d'en éliminer toutes les variables parasites.

Mais, depuis l'époque des enquêtes Hawthorne, l'analyse statistique des données quasi expérimentales et des séries temporelles a été développée ${ }^{57}$. On peut utiliser les données recueillies sur une longue période en vérifiant l'effet des variables mesurées et en opérant les ajustements rendus nécessaires par les changements intervenus au cours du temps dans les facteurs historiques non soumis à la mesure. En particulier, les procédés de l'analyse de régression ont été développés en économétrie. La deuxième expérience dans la série des expériences Hawthorne, celle du first relay experiment, comporte un grand nombre de variables dépendantes et indépendantes que l'on peut transcrire sous une forme quantitative; elles couvrent vingt-trois périodes expérimentales ${ }^{58}$, ce qui permet l'emploi de l'analyse de régression temporelle.

56. R. H. Franke, J. D. KAUl, art. cit. supra n. 6, p. 624-625, donnent les références des principales recherches aux Etats-Unis issues de près ou de loin des expériences Hawthorne.

57. Dans le domaine de l'économétrie auquel ils ont particulièrement recours, les auteurs citent John JoHNSTON, Econometric Methods, New York, McGraw Hill, 1963.

58. Une $24^{*}$ période, qui s'étend du $1^{\text {er }}$ mars 1932 au 8 février 1933 , a été écartée de cette analyse. Durant cette dernière période, les cinq ouvrières ont été renvoyées et remplacées par des ouvrières plus qualifiées mais sans aucune expérience de l'assemblage des relais téléphoniques. Cf. ibid., p. 627, n. 7 . 
Les auteurs ont pu établir une mesure de la production pour le groupe des cinq ouvrières et pour chacune d'elles individuellement, ainsi qu'une mesure de chacune des variables indépendantes. Certaines de ces variables étaient délibérément manipulées par les enquêteurs : les pauses, le nombre d'heures de travail par jour, de jours de travail par semaine, ainsi que l'introduction d'un stimulant financier calculé sur le petit groupe. Les auteurs ont pu également identifier un certain nombre de changements qualitatifs intervenus fortuitement au cours des cinq années de durée des expériences. Les plus intéressants de ces changements sont le remplacement par la maîtrise de deux des ouvrières à l'issue de la septième période (les auteurs rejoignent donc ici l'analyse purement qualitative de Carey) et l'irruption de la crise au début de la quinzième période. L'analyse statistique à laquelle se livrent les auteurs fait usage de toutes ces données pour rechercher directement quelle est la source des différences observées dans la production des ouvrières au cours de la période, et pour déterminer si la variance importante obtenue par l'expérience peut être expliquée en termes quantitatifs.

\section{Les données et la méthode}

Les archives ont été examinées aux établissements Hawthorne ${ }^{59}$. La production y est consignée sous la forme du taux net horaire et hebdomadaire par ouvrière. La qualité de la production est mesurée quant à elle par le nombre d'heures de réparation des relais requises quotidiennement. Le nombre d'heures de travail par jour et par semaine et le nombre de semaines de travail pour chacune des périodes que comporte l'expérience donnent l'emploi du temps normal des ouvrières, réduit par le temps des pauses prévues et des pauses volontaires. Les changements qualitatifs dans les conditions de travail sont représentés par des variables dichotomiques comportant seulement les valeurs zéro et un, et qui concernent des événements significatifs ${ }^{60}$.

Les auteurs ont utilisé pour traiter ces variables les techniques

59. Les archives ont été reproduites sur des microfilms dont les exemplaires ont été déposés à la bibliothèque de l'University of Wisconsin à Milwaukee et à celle du Worcester Polytechnic Institute.

60. Ce sont : 1) le rappel à la discipline par la direction (le remplacement par la direction de deux des cinq ouvrières, l'une des deux remplaçantes jouant le rôle d'un contremaitre de remplacement) ; 2) le début de la dépression économique ; 3) la fourniture de matériaux défectueux pour deux des périodes; 4) le remplacement temporaire sur une base volontaire de l'une des ouvrières; 5) et le passage d'un stimulant financier fondé sur les résultats du Département tout entier à un système propre au petit groupe. 
économétriques adaptées aux séries temporelles, qui permettent de faire porter l'analyse même sur les effets produits par des changements intervenus fortuitement en cours d'expérience. Les auteurs ont en outre utilisé la corrélation sériale qui permet de mesurer l'effet d'autres facteurs historiques plus généraux et de l'écoulement du temps. D'autres techniques permettent de s'assurer de l'indépendance statistique des séries entre elles. Pour plus de clarté, on se concentrera sur l'utilisation des techniques économétriques.

Pour expliquer quantitativement la fluctuation au cours du temps de la production des cinq ouvrières considérées comme un groupe et de chacune d'elles considérée individuellement, l'analyse procède par trois étapes. La première étape examine les corrélations entre les variables au niveau du groupe pour l'ensemble des vingt-trois périodes, et séparément pour les sept périodes antérieures au remplacement des deux ouvrières ne donnant pas satisfaction et pour les seize périodes suivant cette démonstration de rétablissement de la discipline. La seconde étape établit quelles sont les plus importantes régressions au niveau du groupe pour chacune des trois mesures de la production.

\section{Les corrélations au niveau du groupe}

L'analyse des corrélations doit être maniée avec prudence car leur dépendance temporelle ou la corrélation sériale n'ont pas encore été mesurées. Néanmoins, quelques relations simples et perceptibles s'établissent entre les variables mesurant le travail (performance) du groupe et les variables expérimentales, ce qui permet d'obtenir des pourcentages élevés d'explication de la variance $\left(R^{2}\right)$. C'est ainsi que les fluctuations du taux horaire de production peuvent s'expliquer en grande partie (25\% ou plus) par les variables qualitatives du rétablissement de la discipline par la direction et de la dépression économique : en effet, la production horaire du groupe bénéficie selon toute apparence de la décision prise par la direction de remplacer deux ouvrières, et de la dépression. En outre, la réduction des heures de travail hebdomadaire, l'augmentation des pauses, et l'introduction d'un stimulant financier spécifique au petít groupe s'avèrent favorables à l'augmentation du taux de production horaire.

Ce rôle du stimulant financier et des pauses sur la production apparaissent déjà (selon Franke et $\mathrm{Kaul}^{61}$ ) dans Roethlisberger et Dickson $^{62}$. En effet, ces derniers présentent, sans toutefois les analyser,

61. J. JOHNSTON, op. cit. supra n. 57, p. 628 , n. 10 .

62. F. J. Roethlisberger, W. J. Dickson, op. cit. supra n. 9, p. 132, 148. 
les données relatives aux expériences du second relay et du mica-splitting. Dans l'expérience du second relay, la production horaire moyenne par ouvrier est passée de 1634 composants d'unités de relais pour la première période (stimulant financier classique) à 1840 pour la deuxième période (stimulant financier spécifique au petit groupe) et de nouveau 1531 pour la troisième période (retour au stimulant classique). Si l'on prend les périodes les plus anciennes comme périodes de contrôle pour les mêmes ouvrières dans les périodes les plus récentes, l'application du $\mathrm{t}$ de Student montre qu'il n'y a de différence significative qu'entre la première et la deuxième période. Autrement dit, l'introduction du stimulant financier préférentiel s'est traduite par une amélioration de $12,6 \%$ du taux de production. De même, pour les cinq ouvrières de la mica-splitting room, la même analyse statistique indique une augmentation significative et encore plus sensible $(15,5 \%)$ du taux de production horaire, due selon toute apparence à la diminution de la fatigue grâce à des pauses et à la réduction du nombre d'heures de travail. De semblables effets, dus aux stimulants financiers et à la réduction de la fatigue, sont constatés dans des situations diverses par des auteurs comme Bass et Barret ${ }^{63}$, Cherrington et $a l .{ }^{64}$, et bien entendu d'abord Taylor ${ }^{65}$.

Il faut noter au passage que la plupart des variables indépendantes mentionnées ci-dessus sont moins corrélées entre elles qu'avec les variables dépendantes, ce qui augure favorablement d'une explication par analyse multivariée d'une grande partie de la variance de la production horaire par des variables expérimentales connues.

Les corrélations pour deux groupes de périodes (soit les périodes 1 à 7 , antérieures au remplacement par la maîtrise des deux ouvrières et au rétablissement de la discipline, et 8 à 23 , postérieures à ce changement) donnent des résultats intéressants. Pour les premières périodes, l'augmentation du temps de repos (réduisant de $c e$ fait le nombre d'heures de travail) et l'introduction du stimulant financier préférentiel sont favorables à la production. Pour les périodes plus récentes, la dépression économique et la réduction du nombre d'heures de travail ont un effet favorable sur le taux de production horaire. Ces résultats obtenus pour des périodes plus courtes sont en accord avec

63. Bernard M. BASS, Gerald V. BARRETT, Man, Work and Organizations, Boston, Allyn and Bacon, 1972.

64. David J. CherRington, H. Joseph ReIr, William E. SCOTt Jr, « Effects of Contingent and Non-Contingent Reward on the Relationship between Satisfaction and Task Performance ", Journal of Applied Psychology, t. 55, 1971, p. 531.536.

65. Frederick Winslow TAYOR, The Principles of Scientific Management (New York, Harper, 1911), New York, W.W. Norton and Co, 1966. 
les corrélations établies sur la totalité des 23 périodes, pour lesquelles l'effet du rétablissement de la discipline par la direction se fait en outre clairement sentir.

Les corrélations pour le taux hebdomadaire de production ont également été calculées et produisent des résultats quelque peu différents. Les facteurs favorables sont le remplacement de l'ouvrière $n^{\circ} 5$, l'augmentation de l'horaire hebdomadaire de travail, l'augmentation du temps de repos, et l'introduction du stimulant financier calculé d'après les résultats du petit groupe expérimental. A ce niveau des corrélations, les seuls facteurs favorables à la fois au taux horaire et au taux hebdomadaire de production sont les pauses et le stimulant financier préférentiel.

Les corrélations ont aussi été calculées pour la troisième variable liée au travail: le temps requis quotidiennement pour la réparation des pièces produites (mesure inverse de la qualité de la production). La production de mauvaise qualité semble la résultante d'une combinaison de facteurs de deux types : ceux qui jouent sur la qualité de façon pour ainsi dire mécanique (matériaux de mauvaise qualité, présence d'une ouvrière manquant d'expérience) et ceux qui rompent la routine du travail (pauses plus fréquentes, changements plus fréquents dans l'horaire et les conditions de travail).

\section{Les analyses de régression au niveau du groupe}

Chacune des trois variables dépendantes a fait ensuite l'objet au niveau du groupe d'une analyse de régression pas à pas sur les douze variables indépendantes. Les équations de régression sont données dans le Tableau n ${ }^{\circ} 1$ ci-contre (on a laissé de côté pour plus de simplicité les corrections appliquées pour la corrélation sériale et les nouvelles équations de régression qui en résultent).

Pour le taux de production horaire du groupe les variables indépendantes les plus explicatives sont les suivantes d'après le modèle $n^{\circ} 1$ : le rétablissement de la discipline par la direction (qui explique $79 \%$ de la variance), la dépression économique (14\%) et les jours de repos prévus (4\%). Mais il est bien clair que la majeure partie des $97 \%$ de la variance ainsi expliqués résultent du rétablissement de la discipline par la direction, par le remplacement de deux ouvrières du groupe par des ouvrières ayant une production plus élevée, et par la force de l'exemple.

Pour le taux de production hebdomadaire le modèle $\mathrm{n}^{\circ} 2$ indique comme variables indépendantes explicatives dans l'ordre le nombre 


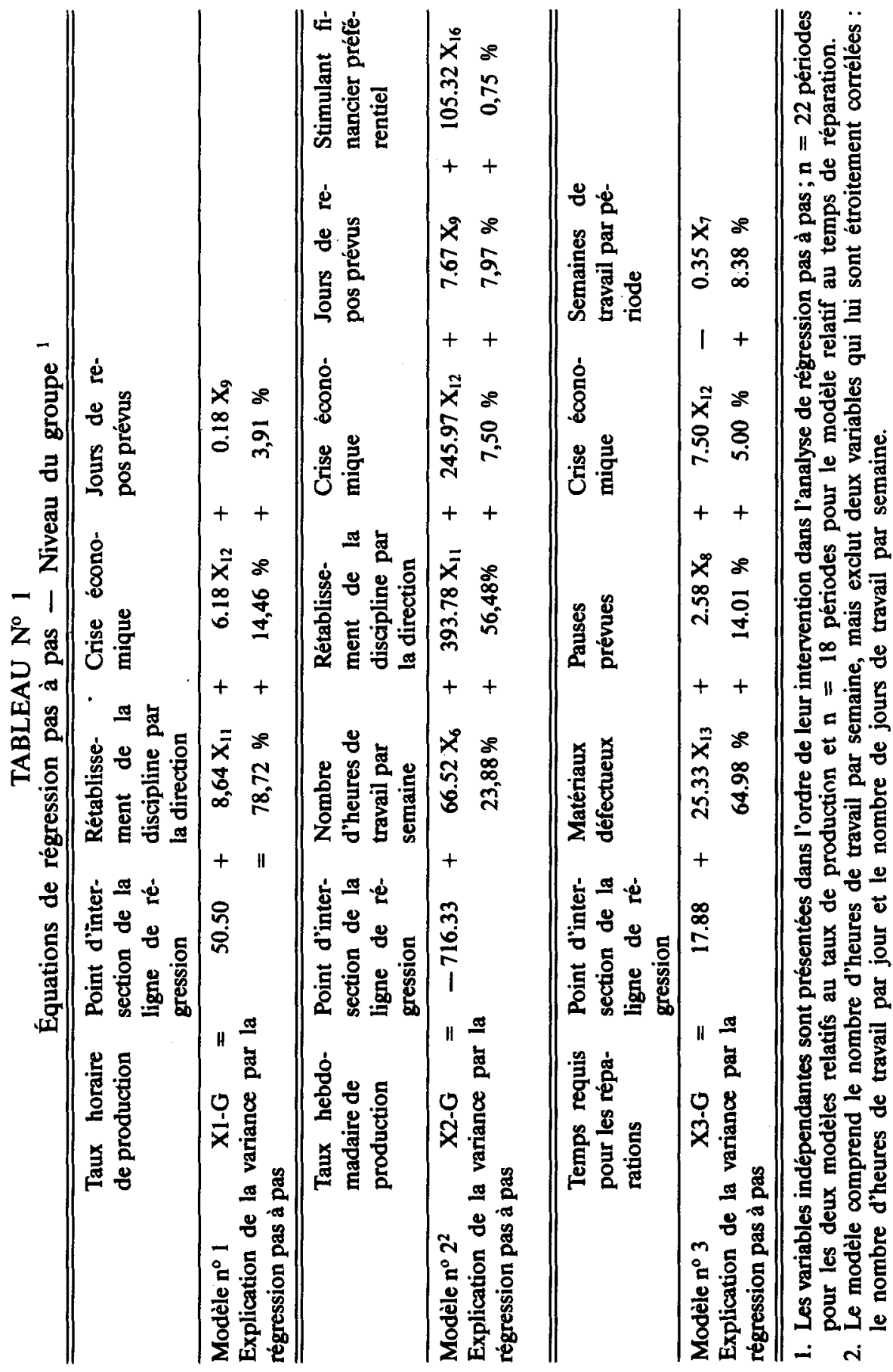


d'heures de travail par semaine (qui ne rend compte cependant que de $24 \%$ de la variance, ce qui la classe au second rang). En deuxième position dans l'analyse de régression, mais de nouveau au premier rang pour l'explication de la variance, on retrouve la discipline rétablie par la direction (56, $48 \%$ ) avec toutefois une prééminence moins accusée que dans le modèle précédent. Suivent la crise économique et les jours de repos prévus $(8 \%)$ et le stimulant financier préférentiel $(1 \%)$ qui joue un rôle négligeable. La totalité de la variance expliquée est à nouveau de $97 \%$.

Pour la mesure inverse de la qualité de la production, fournie par le temps de réparation requis par ouvrière quotidiennement, le modèle $n^{\circ} 3$ donne comme variables indépendantes les plus explicatives d'abord (comme il est normal) la fourniture de composants défectueux (65\%), un plus grand nombre de jours d'arrêt par semaine (14\%), la crise économique $(5 \%)$ et un moins grand nombre de semaines de travail par période d'expérimentation (8\%).

Ainsi, pour chaque variable dépendānte établie au niveau du groupe (cf. Tableau $\mathrm{n}^{\circ} 1$, p. 421), il existe des variables indépendantes quantitatives qui rendent compte de plus de $90 \%$ de la variance. Par exemple, la plus grande partie de la variance dans la quantité produite au niveau du groupe s'explique par le remplacement de deux ouvrières médiocres par deux autres qui ont dès le début atteint des niveaux de production plus élevés. Quant à la variance dans la qualité de la production, elle s'explique par la différence de qualité des composants fournis.

Pour mieux comprendre la signification de ces résultats statistiques et pour mesurer les effets du remplacement sur les ouvrières $1 \mathrm{~A}$ et $1,2 \mathrm{~A}$ et 2 , et l'impact de cette mesure exemplaire du rétablissement de la discipline sur les ouvrières 3,4 et 5 , les analyses de régression ont été répétées au niveau des individus, soit $5+2$ ouvrières.

En appliquant aux ouvrières prises individuellement les modèles qui sont à la fois les plus explicatifs au niveau du groupe et les plus efficaces au niveau individuel, on obtient (après correction) pour le taux horaire de production $87 \%$ d'explication de la variance, $92 \%$ pour le taux hebdomadaire, et $75 \%$ pour le temps de réparation, soit un peu moins que les $94 \%$, $96 \%$ et $93 \%$ obtenus après correction pour le groupe tout entier.

Ainsi les données mêmes recueillies par Roethlisberger et Dickson permettent d'établir clairement que le taux de production sous ses différentes formes s'explique de façon satisfaisante précisément par le type de variables qu'ils souhaitaient écarter. Ce sont des facteurs classiques comme les pauses, la discipline, le stimulant financier (facteurs de nature très " taylorienne ") joints à des changements de conjoncture 
(également exclus par Roethlisberger et Dickson) qui fournissent les meilleures explications. Par exemple, la crise économique n'est évoquée par les auteurs de l'enquête et par la plupart des commentateurs que dans son rôle d'élément perturbateur mettant prématurément fin à l'expérience. Franke et Kaul interprètent la relation positive constatée entre la production et la crise en soulignant que cette dernière agit en fait comme un stimulant : comme tous les ouvriers, chacun des participants à l'enquête Hawthorne tient à conserver son emploi et augmente de ce fait son taux de production.

\section{IV. - QUE FAIRE DES ENQUÊTES HAWTHORNE? \\ OU IDÉOLOGIE ET RATIONALITÉ DANS LES SCIENCES DE L'HOMME}

Les deux examens critiques des expériences Hawthorne que nous avons retracés en détail sont évidemment entièrement différents dans leurs données et dans leurs démarches. Carey procède, en effet, à une confrontation purement interne des passages d'entretiens et d'observations (données qualitatives) et accessoirement des données statistiques de production (données quantitatives) sous la forme dans laquelle elles ont èté publiées. Il conclut, au terme de cet examen, à l'incohérence des expériences Hawthorne. Selon une logique qu'on peut qualifier de néo-positivisme expérimentaliste, Carey passe de ce constat à une conclusion radicale : la nullité scientifique de tout le mouvement des relations humaines.

Franke et Kaul procèdent différemment. Ils remontent aux données d'archives non publiées sur la production des cinq ouvrières de la deuxième expérience (first relay experiment) et, à l'aide de méthodes d'analyse de corrélations simples et surtout de régression temporelle, établissent (plus radicalement encore que Carey) que ces données statistiques sur les fluctuations de la production des ouvrières s'avèrent, sans équivoque, favorables aux thèses de l'organisation scientifique du travail (O.S.T.) d'inspiration taylorienne. Or $œ$ sont ces thèses que l'équipe des expériences Hawthorne, principalement sous l'influence d'Elton Mayo, entendait combattre.

Toutefois Franke et Kaul se montrent beaucoup plus nuancés que Carey dans leur jugement sur les expériences Hawthorne. Ils ne les condamnent que pour ce qui relève de leur objectif initial explicite : établir empiriquement le bien-fondé de la proposition selon laquelle l'amélioration des relations humaines est la seule voie qui puisse conduire à des niveaux de production plus satisfaisants. Mais ils 
soulignent aussitôt après que bien d'autres thèmes, déjà présents dans les expériences Hawthorne, ont été explorés par la suite par le mouvement des relations humaines. Sur la base de l'idée centrale du primat des relations humaines, des notions dérivées telles que l'effet de groupe, le moral, le freinage, la résistance au changement, ont inspiré jusqu'au milieu des années cinquante des recherches fructueuses (voir B. Mottez déjà cité pour le développement et l'articulation de ces thèmes de recherches ${ }^{66}$ ).

Cette conclusion parait historiquement plus satisfaisante que celle avancée par Carey. En décrétant la nullité des expériences Hawthorne et de facto, du mouvement des relations humaines qui s'en est tellement réclamé, Carey ne peut que constater comme une énigme insoluble l'incontestable succès ultérieur du mouvement : sa " history" demeure impuissante devant la "story" qui résiste malgré ses efforts. Franke et Kaul sont plus subtils. Il y aurait eu d'après eux déplacement des buts initiaux du mouvement des relations humaines. On serait passé de l'amélioration des taux de production à la recherche de l'amélioration de facteurs plus fins, globalement subsumés sous le terme de "satisfaction au travail" ou de "climat social de l'entreprise". Mais cette interprétation suppose elle aussi que les expériences Hawthorne soient plus ou moins négligées au profit de nouveaux objets de recherche.

Le paradoxe est qu'il n'en est rien. Les nouveaux sujets de recherche désignés pertinemment par Mottez ainsi que par Franke et Kaul sont adoptés par tous les auteurs jusque vers 1950 au nom de la scientificité postulée et constamment réaffirmée des expériences Hawthorne. On pourrait encore expliquer ce paradoxe en s'inspirant d'exemples appropriés, empruntés à l'histoire ou à la sociologie de sciences autres que les sciences de l'homme. Bien des expériences non concluantes ou apparemment "négatives", en supposant que la notion avancée par Popper de la réfutation (" falsification " dans la traduction anglaise) des théories scientifiques puisse être retenue dans sa simplicité drastique ${ }^{67}$, ont pu donner naissance par la suite à des développements fructueux.

Mais le cas des expériences Hawthorne et des publications qui leur sont associées est entièrement différent. En effet, ni Carey, ni même Franke et Kaul n'auraient pu mener à bien leur œuvre consciencieusement destructrice d'historiens dévoreurs de mythes sans un fait tout à fait singulier. Ce fait est tout simplement le suivant. Ce sont les auteurs

66. B. MotTEz, op. cit. supra n. 1.

67. La discussion classique sur ce point entre Popper, Kuhn, Lakatos et Feyerabend se trouve dans Imre LAKaToS, Alan Musgrave, Criticism and the Growth of Knowledge, Cambridge, Cambridge University Press, 1970. 
mêmes des expériences jugées ultérieurement fautives qui leur ont fourni tous les éléments de leur propre condamnation.

Comment expliquer dans ce contexte l'assurance paisible des auteurs gravitant dans les années 1935-1940 autour des expériences Hawthorne? Une première hypothèse, évidemment peu satisfaisante, est celle de l'incompétence. Je préfère avancer l'hypothèse de la certitude que confère l'idéologie. Il faut toutefois préciser que je n'entends pas ici par « idéologie " un mouvement d'idées entièrement passionnel, échappant à toute rationalité et, a fortiori, à la scientificité. Tout au contraire, les deux idéologies qui nous intéressent ici, l'organisation scientifique du travail et le mouvement des relations humaines, me paraissent fondées l'une comme l'autre sur des noyaux de scientificité d'extension limitée, difficilement contestables à l'intérieur même de ces limites. Un exemple de tels noyaux serait, chez Taylor ou dans l'O.S.T. ultérieur, le gain de temps obtenu par la décomposition des travaux simples en mouvements élémentaires, ou l'effet stimulant des salaires au rendement. Il est à signaler que, pour l'O.S.T., les noyaux de scientificité se situent principalement au niveau des individus. L'élément proprement idéologique, dans le cas du taylorisme ou de l'O.S.T., consiste, sur la base de cette scientificité à portée limitée, à élaborer et présenter par extrapolation illégitime des solutions prétendûment scientifiques aux problèmes majeurs de la société industrielle, c'est-à-dire principalement aux conflits du travail et aux litiges sur les rémunérations. Les essais d'introduction du taylorisme, tant aux États-Unis qu'en France, ont généralement été accompagnés par une extension spectaculaire des conflits sociaux ${ }^{68}$ qu'il était censé résoudre, en raison de l'extension illégitime à des groupes sociaux, des entreprises ou des organisations entières de conclusions valides principalement (voire exclusivement) au niveau des individus.

Le mouvement des relations humaines présente, quant à lui, des caractéristiques symétriques mais inversées. Ses noyaux de scientificité ne sont pas dérivés de l'ergonomie ou de la psychologie économique, mais de l'observation ethnologique et de l'entretien clinique ${ }^{69}$. Ils se

68. Sur la France, Patrick Fridenson, " Un toumant taylorien de la société française (1904-1918) », Annales. E.S.C., 5, sept.-oct. 1987, p. 1031-1060. Sur Taylor et les États-Unis, Daniel Nelson, Frederick W. Taylor and the Rise of Scientific Management, Madison, University of Wisconsin Press, 1980 et « Le taylorisme dans l'industrie américaine 19001930 », in Le Taylorisme, sous la dir. de Maurice DE MONTMOLLIN, Olivier PASTRE, Paris, La Découverte, 1984, p. 13-32.

69. D'après L. URwick, op. cit. supra n. 11, p. 12 : “Mayo was a psychiatrist, not a sociologist [...]. The basis of his thinking was that many of our troubles [...] are in fact 
situent donc ailleurs que là où ils se situent dans le taylorisme : audessous de l'individu conscient et calculateur, dans les motivations inconscientes des travailleurs, et au-dessus de ce même individu, au niveau de la solidarité du groupe et des comportements apparemment paradoxaux qu'elle inspire. Le comportement du «freinage » est un bon exemple de noyau de scientificité propre au mouvement des relations humaines. En effet, il consiste de facto en une reprise et une inversion de l'un des noyaux centraux du taylorisme: l'efficacité au niveau des individus du salaire au rendement.

On comprend mieux pourquoi, contrairement à ses affirmations répétées, le mouvement des relations humaines n'a, en fait, jamais réellement supplanté le taylorisme ni l'O.S.T. ${ }^{70}$. Aucun des deux systèmes ne pouvait « logiquement » renverser l'autre. En effet, la partie proprement idéologique (dérivant d'une extension illégitime) de chacune des deux constructions échappe par définition à toute procédure de réfutation. On peut même, avec Bramel et Friend ${ }^{71}$ et sans obligatoirement admettre leur a priori marxiste, souligner la convergence des deux constructions idéologiques (l'une fondée sur le modèle de l'individu rationnel, l'autre sur le groupe restreint) dans une représentation commune de la classe ouvrière comme soumise, passive (quoique nettement moins chez Mayo que chez Taylor), prête aux manipulations et incapable d'une action collective d'envergure. Qui plus est, les parties réfutables (ou noyaux de scientificité) ne se réfèrent pas au même niveau de la réalité sociale et ne sont donc pas en rapport de contradiction. Cette articulation de l'idéologie et de la scientificité - présentée ici à titre d'hypothèse - apparaît plus plausible dans les sciences de l'homme que dans les sciences mathématiques, physiques ou biologiques. Ces dernières assortissent, en effet, les propositions (ou les lois) qu'elles énoncent de conditions très précises quant aux limites de leur validité. Les sciences de l'homme ont au contraire tendance à avancer d'emblée soit des propositions universelles sous forme de "lois de succession " (après $A, B$ et nécessairement $C$ ), soit des propositions conditionnelles (si $A$, alors $B$ ), mais le plus souvent d'un très grand niveau de généralité. Il est donc tentant et facile dans une structure de cet ordre de franchir

due to conflicts within ourselves. "Sur le rôle de la psychiatrie et de l'ethnologie dans la formation d'Elton Mayo, voir, outre Urwick, William F. WHYTE, "MAYO, Elton », in Intemational Encyclopedia of the Social Sciences, David L. Sills, ed., New York, CollierMacmillan, 1968, vol. 10, p. 82-83. E. SARIN, art. cit. supra n. 6, p. 12, signale un article de E. MAYo, « Psychiatry and Sociology in Relation to Social Disorganization ", American Jownal of Sociology, déc. 1937.

70. Sur $\propto$ point, of. M. DE MONTMOllin, Le Taylorisme d visage humain, Paris, Presses de France, 1981, et Le Taylorisme, op. cit. supra n. 68.

71. D. Bumet, R FrIEND, art. cit. supra n. 6. 
des limites de validité d'une proposition, de la détourner à des fins idéologiques - en bref, de faire d'une proposition à scientificité limitée une vérité universelle. Le taylorisme franchit ce (mauvais) pas en prétendant instaurer la paix sociale sur le seul fondement d'une base équitable de la rémunération du travail, celle du rendement. Réciproquement, le mouvement des relations humaines franchit les limites de validité de ses observations sur l'effet de groupe sous toutes ses formes en prétendant les relier quantitativement à la production.

La place logique et le rôle historique des expériences Hawthorne se comprennent mieux dans une telle perspective. Logiquement parlant, il apparait clairement, comme le disent Franke et Kaul, qu'il y a eu en fait déplacement des buts initiaux des expériences (l'explication des fluctuations de la production) pour la recherche de buts plus subtils. Toutefois les auteurs, comme éblouis par leur découverte de la solidarité du petit groupe ( $2^{e}$ phase ou first relay experiment), ont maintenu leurs prétentions antérieures à expliquer les fluctuations de la production, cela au prix de distorsions constantes dans l'interprétation qualitative et quantitative de leurs propres résultats. Par la suite, la référence à ces résultats ainsi malmenés est demeurée constante parce qu'elle fournissait une apparence crédible de scientificité à une philosophie sociale globale et à des hypothèses de recherche intéressantes : pour fournir cette caution, les résultats devaient être à la fois globalement invoqués et ne jamais être sérieusement réanalysés. C'est bien apparemment ce qui s'est produit. Il resterait à examiner, dans la ligne adoptée par Bramel et Friend mais de façon historiquement mieux adaptée, le contexte social et les facteurs sociaux contemporains et compatibles (sinon explicatifs au sens fort) du mouvement des relations humaines et de son rapprochement dans la pratique avec le taylorisme ${ }^{72}$. Nouveaux besoins du patronat en matière de gestion du personnel, correspondant vraisemblablement à une nouvelle phase du capitalisme, groupes professionnels respectifs (ingénieurs, ergonomes ou psychologues, ethnologues et sociologues), enjeux de part et d'autre, devraient notamment être étudiés. Cela serait évidemment l'objet d'un tout autre travail.

Bernard-Pierre LÉCUYER, Groupe d'Étude des méthodes de l'analyse sociologique (CNRS/Université Paris IV) Maison des sciences de l'homme.

72. P. FRIDENSON, art. cit. supra n. 68. 